\title{
A Wideband Channel Model for Intravehicular Nomadic Systems
}

\author{
François Bellens, ${ }^{1,2}$ François Quitin,, ${ }^{1,3}$ Jean-Michel Dricot, ${ }^{1}$ François Horlin, ${ }^{1}$ \\ Aziz Benlarbi-Delai,, ${ }^{2}$ and Philippe De Doncker ${ }^{1}$ \\ ${ }^{1}$ Optics, Photonics, Electromagnetism, Radio-Communications and Acoustics (OPERA) Department, \\ Université Libre de Bruxelles (ULB), Av. F.D. Roosevelt 50, 1050 Brussels, Belgium \\ ${ }^{2}$ Laboratoire d'Electronique et Electromagnétisme (L2E), Université Pierre et Marie Curie-Paris 6 (UPMC), Place Jussieu 4, \\ 75252 Paris, France \\ ${ }^{3}$ Information and Communication Technologies, Electronics and Applied Mathematics (ICTEAM), Electrical Engineering, \\ Université Catholique de Louvain (UCL), Place du Levant 2, 1348 Louvain-la-Neuve, Belgium
}

Correspondence should be addressed to François Bellens, fbellens@ulb.ac.be

Received 19 May 2011; Accepted 15 June 2011

Academic Editor: Dau-Chyrh Chang

Copyright (C) 2011 François Bellens et al. This is an open access article distributed under the Creative Commons Attribution License, which permits unrestricted use, distribution, and reproduction in any medium, provided the original work is properly cited.

The increase in electronic entertainment equipments within vehicles has rendered the idea of replacing the wired links with intravehicle personal area networks. Ultra-wideband (UWB) seems an appropriate candidate technology to meet the required data rates for interconnecting such devices. In particular, the multiband OFDM (MB-OFDM) is able to provide very high transfer rates (up to $480 \mathrm{MBps}$ ) over relatively short distances and low transmit power. In order to evaluate the performances of UWB systems within vehicles, a reliable channel model is needed. In this paper, a nomadic system where a base station placed in the center of the dashboard wants to communicate with fixed devices placed at the rear seat is investigated. A single-input singleoutput (SISO) channel model for intra-vehicular communication (IVC) systems is proposed, based on reverberation chamber theory. The model is based on measurements conducted in real traffic conditions, with a varying number of passengers in the car. Temporal variations of the wireless channels are also characterized and parametrized. The proposed model is validated by comparing model-independent statistics with the measurements.

\section{Introduction}

Interest in wireless personal area networks (WPANs) for intravehicle communications (IVCs) has significantly increased. In addition to sensor networks used to perform vehicle maintenance (tire pressure, oil levels,...), WPANs are considered as a possibility for interconnecting entertainment equipment within the vehicle. Currently, electronic devices (e.g., DVD players, positioning devices, mobile phones,...) are connected using wired or fiber-optic links. The replacement of those wired links by wireless networks will decrease installation cost and time and increase the network flexibility. In this paper, a nomadic system is investigated: the base station is placed in the center of the dashboard and wants to communicate with fixed devices placed at the rear seat.

To enable high connectivity, a solution must be found that provides sufficient data rate, has low power consumption, and if possible, has low cost. One potential candidate is the Ultra-wideband (UWB) technology, and in particular the Multi-Band Orthogonal Frequency Division Multiplexing (MB-OFDM). The MB-OFDM is standardized by the European Computer Manufacturers Association (ECMA) under the standard ECMA-368 [1]. The standard allows communication in the 3.1 to $10.6 \mathrm{GHz}$ frequency band and defines different transmission modes to support high data rates (up to $480 \mathrm{Mbps}$ ). Another emerging solution that can offer sufficient data rate is the $60 \mathrm{GHz}$ communication systems, which operates in the $57-66 \mathrm{GHz}$ band. Currently, different alliances and consortiums have published their own standard or specification such as the ECMA [2], the Wireless HD consortium [3], and the Wireless Gigabit Alliance [4].

In order to evaluate the performance of MB-OFDM systems within vehicles, a reliable UWB channel model is needed. This model has to include the specific propagation characteristics of IVC environments. Several studies have 
started to investigate the IVC-UWB channel [5-11]. In [5], the authors present experimental results on the rms delay spread for two types of vehicles with different numbers of passengers and with different antenna's locations. In [6], the UWB impulse channel and in particular the rms delay spreads are investigated with and without passengers. Results on the interference outside the car were presented showing that the inference is relatively small. Spatial characteristics of the IVC channel are investigated in [7]. In [8], the authors extract some large- and small-scale parameters from measurements in an empty and an occupied car. The effect of the type of car on these parameters was further studied in [9]. In [10], the authors present a performance comparison between the UWB MB-OFDM systems and the $60 \mathrm{GHz}$ technology. Finally, in [11], measurements were carried out in the $60 \mathrm{GHz}$ band, with and without passengers, and with closed and open doors.

However, none of these papers propose a channel model that suits well for the scenario investigated here and that can be easily implemented for performance evaluation. In $[12,13]$, the propagation parameters inside a car were investigated and comparison between two environments were presented. Based on those results, more measurements were taken in real traffic conditions in different environments and with another car. The aim of this paper is to complete the single-input single-output (SISO) channel model for IVC environment in this nomadic scenario. The channel model is based on measurements taken in real traffic condition and for a varying number of passengers (from 1 to 4 passengers). The paper is organized as follows. In Section 2, the measurement setup, a preliminary study of the channel's first-and secondorder statistics and a comparison with the reverberation chambers are presented. Section 3 presents the structure of the SISO channel model, the channel model parameter extraction, and the temporal behaviour of the channel. In Section 4, it is briefly explained how the proposed model can be implemented. Section 5 validates the proposed model, and finally Section 6 concludes the paper.

\section{Experimental Investigation}

2.1. Experimental Setup. The measurements were carried out inside a common urban Peugeot 207 car $(4030 \mathrm{~mm} \times$ $1970 \mathrm{~mm} \times 1465 \mathrm{~mm})$. Three different measurement sites were chosen in order to compare the influence of different environments on the IVC propagation. The first site was a large avenue with two lanes in each direction separated by a central cleared area. The second measurement site consisted of a set of narrow streets with one-way traffic and streets with one lane of traffic in each direction. The third measurement site was a highway, with three lanes in each direction. The measurements were carried out in normal traffic condition (traffic lights, passing, stopping,...) and at different speeds depending on the environments. For the first environment, the average speed was $50 \mathrm{~km} / \mathrm{h}, 30 \mathrm{~km} / \mathrm{h}$ in the narrow streets and $120 \mathrm{~km} / \mathrm{h}$ on the highway.

For each environment, measurements were made by varying the number of passengers from 1 to 4 people. The

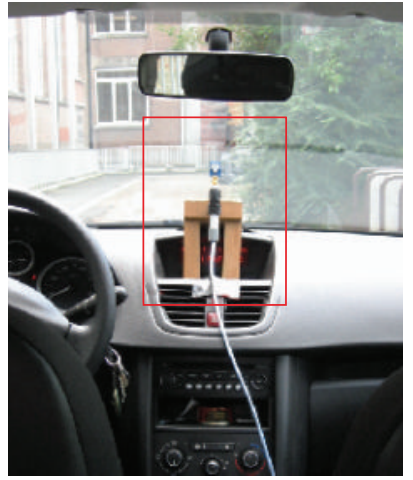

(a) Front Antenna (TX). The TX was placed in the center of the dashboard

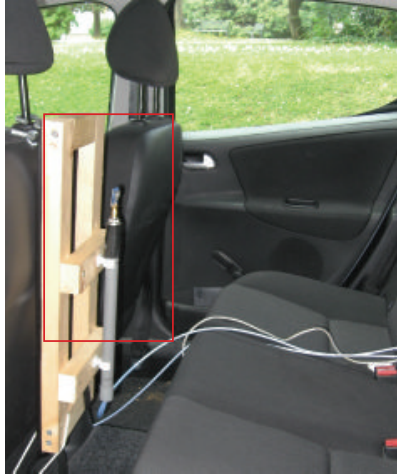

(b) Backside Antenna (RX). The RX was placed at a fixed position behind the driver's seat

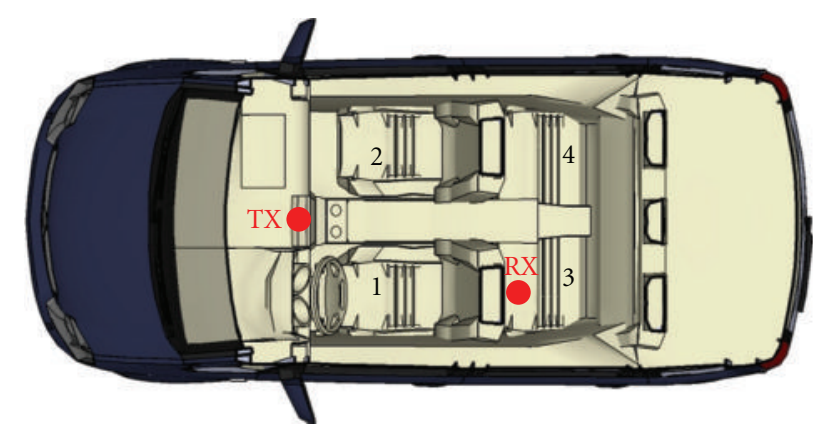

(c) Experimental Setup. Measurements were made by varying the number of passengers from 1 to 4 people. The seats at the front were first filled, then the seats at the rear

FIgURE 1: Pictures and top view of the experimental setup.

seats at the front of the vehicle were first filled, then the seats at the rear. To be the most realistic, the passengers' movements were like in a normal situation for car travel. As mentioned previously, a nomadic system was investigated: the transmitter was placed in the center of the dashboard and the receiver was placed behind the driver's seat. There was no line of sight between the two antennas, and the distance between the antennas was approximately one meter. The location of the antennas and the different positions of the passengers are shown in Figure 1. For each configuration of passengers, ten sets of measurements were taken.

The channel frequency responses were collected with a Rohde \& Schwarz ZVL vector network analyzer (VNA), with omnidirectional broadband SMT3TO10M SkyCross antennas. The VNA was placed in the trunk of the car to avoid any interaction with the measurements. The measurements were made in the first frequency band of the MB-OFDM standard [1], from 3.168 to $4.752 \mathrm{GHz}$, with a $3 \mathrm{MHz}$ frequency step. The transmitted power was set to $4 \mathrm{dBm}$, and the IF-Bandwidth was set to $10 \mathrm{kHz}$. For each set of measurements, 200 sweeps were taken with a sweep time of $0.175 \mathrm{sec}$. The frequency responses were transformed into impulse responses by applying an inverse fast Fourier transform (weighted with a Hamming window to reduce sidelobes). 


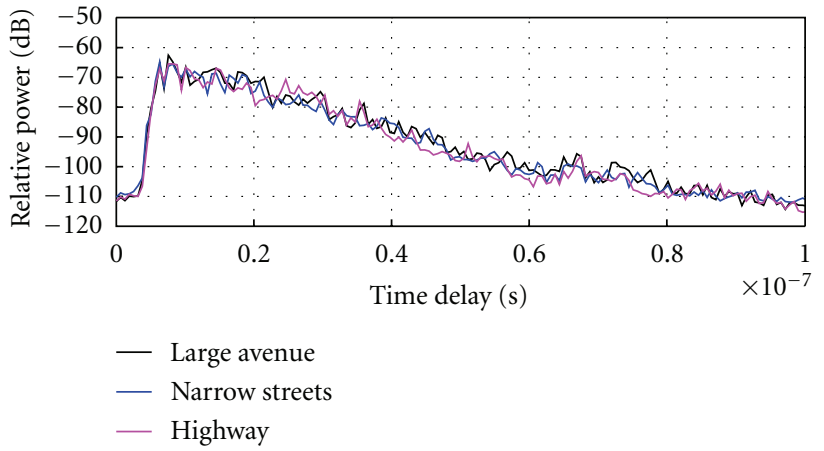

(a) Comparison of the Power Delay Profiles between the different environments for 3 passengers. No significant differences can be observed between the different environments

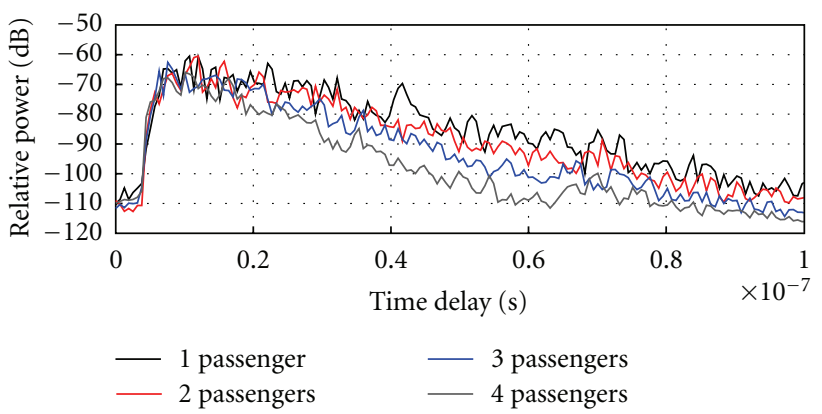

(b) Comparison of the Power Delay Profiles between different number of passengers in the Large Avenue environment. A faster attenuation occurs when the number of passengers is increased

Figure 2: Comparison of the Power Delay Profiles for the different environments (a) and for different number of passengers (b).

2.2. Observations. Comparisons of Power Delay Profiles (PDPs) for the different environments and different number of passengers are shown in Figure 2. It can be seen that the PDPs decrease linearly in $\mathrm{dB}$, as can be observed in diffuse channels [14] or in reverberation chambers [15]. No significant differences can be observed between the PDPs for the different environments, but a faster attenuation occurs when the number of passengers is increased. This observation is confirmed by the rms delay spread values reported in Figure 3(a), where only paths with relative power less than $-30 \mathrm{~dB}$ compared to the peak value are considered. As can be observed, values of the rms delay spread decrease with the number of passengers. The mean values are around $9 \mathrm{~ns}$ for 1 passenger and around $5 \mathrm{~ns}$ for 4 passengers. For each scenario, the standard deviation is around $0.4 \mathrm{~ns}$.

In $[12,13]$, the rms delay spread was around $7 \mathrm{~ns}$ for 1 passenger and decreased around $4.5 \mathrm{~ns}$ for 4 passengers. Those values are comparable with those presented in Figure 3(a). The small differences between the measurements can be explained by the fact that the cars used for the measurement were not the same. In $[12,13]$, the car was a station wagon, while in this study, the car is a supermini. The different IVC environments' sizes and the different reflective properties of the windows can be some explanations. The

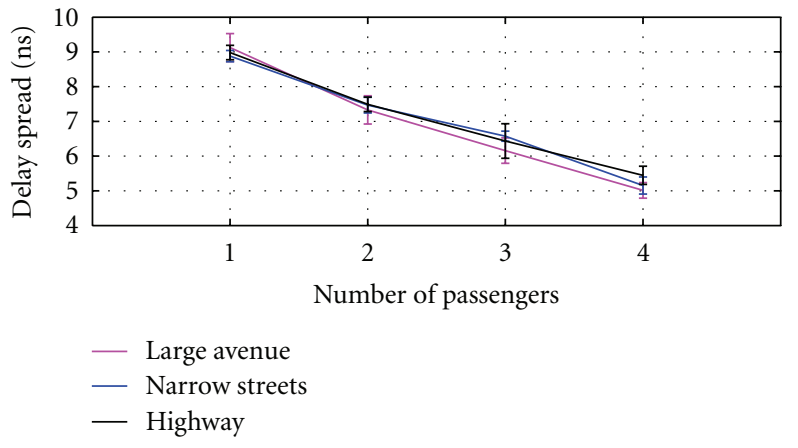

(a) Rms delay spread versus the number of passengers

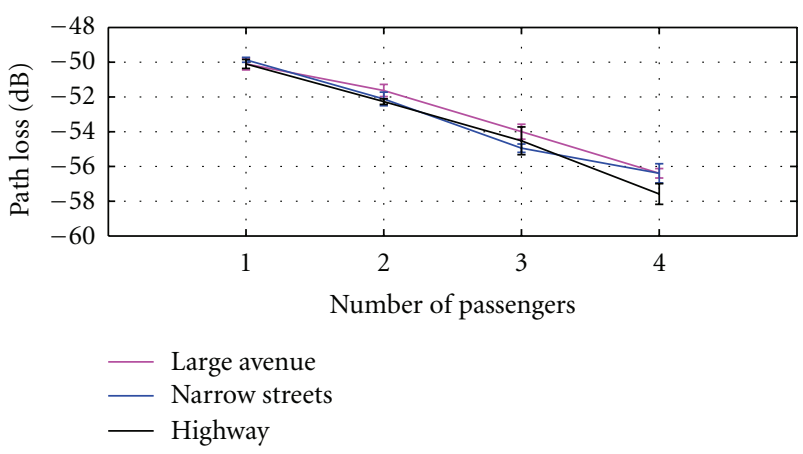

(b) Path loss versus the number of passengers

Figure 3: Rms delay spread (a) and path loss (b) in function of the number of passengers. For both parameters, values decrease with the number of passengers and are quite similar for all the environments.

different antenna's location can also explain the small differences.

In Figure 3(b), the narrowband path loss for all scenarios is presented. It can be seen that the path loss also depends on the number of passengers in the car. Again, the values are quite similar for all types of environments. The mean values are around $-50 \mathrm{~dB}$ for 1 passenger and around $-57 \mathrm{~dB}$ for 4 passengers. For each scenario, the standard deviation is around $0.5 \mathrm{~dB}$. For each number of passengers and for every environment, values of the rms delay spread and the path loss are distributed according to normal distributions.

In order to statistically compare the results of the different environments, Student's $t$-tests were performed between measurements of each environment and a global set including the measurements of all environments. The $t$-tests revealed that there are no significant differences between the different environments and the global set, meaning that there are no statistical differences between the environments. Mean values and standard deviations of the rms delay spreads and path loss for the global set including the measurements of all the environments are given in Table 1.

The results indicate that the propagation inside the car is independent of the environment outside the car. Only what happens inside the car (e.g., number of passengers, size of car, etc.) will have an impact on the IVC channel so confirming $[12,13]$. In the following sections, the channel statistics and 
TABle 1: Mean values and standard deviations of the rms delay spread and path loss for the global set including the measurements of all the environments.

\begin{tabular}{lllll}
\hline \multirow{2}{*}{ Number of passengers } & \multicolumn{3}{c}{ Delay spread (nsec) } & \multicolumn{2}{c}{ Path loss $(\mathrm{dB})$} \\
& Mean & Std. & Mean & Std. \\
\hline 1 & 9 & 0.29 & -50 & 0.38 \\
2 & 7.47 & 0.41 & -51.95 & 0.42 \\
3 & 6.36 & 0.38 & -54.42 & 0.65 \\
4 & 5.18 & 0.39 & -56.76 & 0.73 \\
\hline
\end{tabular}

parameters will be extracted from the global set of measurements, without separating the different environments.

From an electromagnetic point of view, the intravehicular environment can be considered as a loaded cavity or a loaded reverberation chamber (RC) $[15,16]$ with low $Q$-factor. The PDPs presented in Figure 2 indicated that the car acts like the RC as a confined and highly reflective environment. The presence of the seats, plastic, and other stuff in the car can be seen as the absorber while the passengers can be considered like a stirrer moving randomly that interacts with the radiated energy (a stirrer is a mechanical blade placed within the reverberating chamber).

In Figures 3(a) and 3(b), the rms delay spreads and the path losses dependences with the the number of passengers were presented. In both cases, the parameters decrease with the number of passengers. Those dependences indicate that, in this frequency range, the human body does not reflect all the energy but absorbs a nonnegligible amount of energy, as expected. This means that the human body acts also like absorbers that lower the $Q$-factor.

\section{IVC SISO Channel Model}

3.1. Channel Model. In tapped-delay line models [17], the channel impulse response $h(\tau, t)$ is given by

$$
h(\tau, t)=\sum_{k=1}^{N} \alpha_{k}(t) \delta\left(\tau-\tau_{k}\right)
$$

where $\tau_{k}$ denotes the delay of each tap $k$ with complex amplitude $\alpha_{k}$ and $\delta(\tau)$ denotes the Dirac function.

In [18], the author expresses the wireless channel in a loaded reverberation chamber as the sum of two components: the direct component and a component associated with the energy that interacts with the stirrer. This latter is Rayleigh distributed, and it can be interpreted as the diffuse component. The proposed model for IVC channels is based on this approach. The impulse response is written as the sum of a coherent component $h_{c}$ and a diffuse component $h_{d}$ :

$$
h(\tau, t)=h_{c}(\tau)+h_{d}(\tau, t)
$$

where, by denoting $\langle\cdot\rangle_{t}$, the average over time

$$
h_{c}(\tau)=\langle h(\tau, t)\rangle_{t}
$$

For a given delay $\tau$, from (2) and (3), the diffuse power is

$$
\begin{aligned}
\left\langle\left|h_{d}(\tau, t)\right|^{2}\right\rangle_{t} & =2 \operatorname{Var}[\operatorname{Re}(h(\tau, t))]_{t} \\
& =2 \operatorname{Var}[\operatorname{Im}(h(\tau, t))]_{t},
\end{aligned}
$$

where $\operatorname{Var}[\cdot]_{t}$ denotes the variance over time and, $\operatorname{Re}(\cdot)$ and $\operatorname{Im}(\cdot)$ denote the real and imaginary part, respectively.

For each tap, $|h(\tau, t)|$ has a Rice distribution characterized by a Rice factor $K(\tau)$ :

$$
K(\tau)=\frac{\left|h_{c}(\tau)\right|^{2}}{\left\langle\left|h_{d}(\tau, t)\right|^{2}\right\rangle_{t}} \triangleq \frac{\nu(\tau)^{2}}{2 \sigma(\tau)^{2}}
$$

where $\nu(\tau)$ and $\sigma(\tau)$ are the parameters of the Rice distribution.

Using (3) and (4), the powers of the coherent and the diffuse components for each scenario are extracted. Figure 4 presents the case with two passengers. In [14], the authors present an exponential model that suits well to describe the diffuse component. In the delay-domain, the PDP of this model for an infinite bandwidth, $\psi_{d}(\tau)$, is given by

$$
\psi_{d}(\tau)= \begin{cases}0 & \text { if } \tau<\tau_{d}, \\ \frac{\alpha_{d}}{2} & \text { if } \tau=\tau_{d}, \\ \alpha_{d} e^{-B_{d}\left(\tau-\tau_{d}\right)} & \text { if } \tau>\tau_{d},\end{cases}
$$

where $\tau$ represents the delay, $\tau_{d}$ is defined as the base delay, $\alpha_{d}$ is the maximum power, and $B_{d}$ is the decay factor. Here, $B_{d}$ is equal to the inverse of the rms delay spread of the diffuse component and $\alpha_{d}$ is given by

$$
\alpha_{d}=\frac{B_{d} P_{d}}{\mathrm{BW}},
$$

where $P_{d}$ is the total power of the diffuse component and BW is the bandwidth (given in Section 2.1). The last parameter, $\tau_{d}$, is found using a least squares estimator [14]. The approximation of the diffuse component with the model (6) is shown in Figure 4. For each sets of measurements, the parameters of the diffuse component (6) as a function of the number of passengers were extracted. The mean values and the standard deviations are given in Table 2. It can be observed that the values for the rms delay spread of the diffuse component decrease with the number of passengers.

For the same case with two passengers, the measured Rice factor $K(\tau)$ of all the measurements' sets is shown in Figure 5(a). As can be observed, a linear regression matches the mean value decrease. The distribution of all the mean values around the linear regression is normal (in $\mathrm{dB}$ ), with a standard deviation $\varsigma_{1}$ of about $3 \mathrm{~dB}$ for all scenarios. Figure 5(b) presents a detailed view of the measured $K$ factors. As it can be observed, the measured $K$-factors present, for each delay, a normal distribution (in $\mathrm{dB}$ ) around the mean value. The mean standard deviation, represented by $\varsigma_{2}$ on Figure $5(\mathrm{~b})$, is about $3.5 \mathrm{~dB}$. The mean $\overline{K(\tau)}$ factor model, in $\mathrm{dB}$, is given by

$$
\overline{K(\tau)}=K_{1} \cdot \tau+K_{0}+\chi_{1},
$$




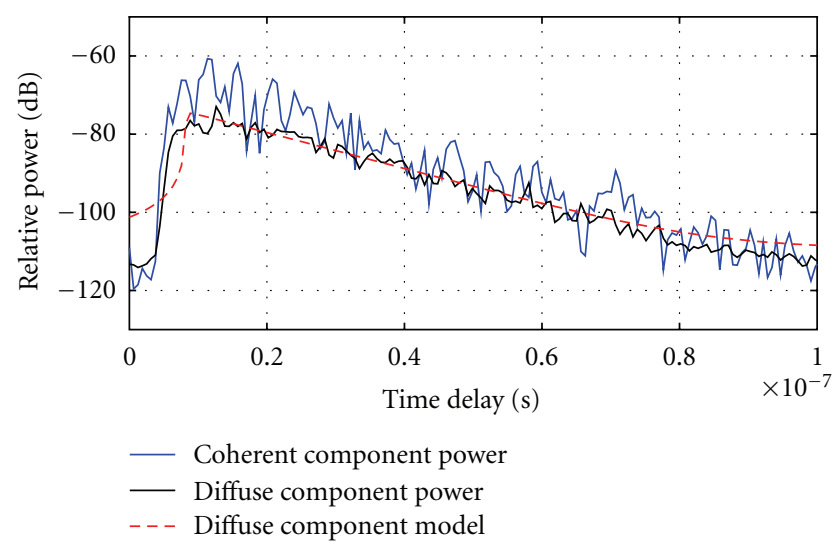

FIGURE 4: Extraction of the powers associated with the coherent (blue line) and diffuse (black line) components. The red dotted line presents the exponential model used to characterize the diffuse component. The example presented here is the case with two passengers.

where $\chi_{1}$ has a normal distribution $N\left(0, \varsigma_{1}\right)$. The $K(\tau)$ factor model in $\mathrm{dB}$, for one set of measurement, is then given by

$$
K(\tau)=\overline{K(\tau)}+\chi_{2}
$$

where $\chi_{2}$ has a normal distribution $N\left(0, \varsigma_{2}\right)$.

The experimental parameters used to characterize the diffuse component and the $K$-factors are summarized in Table 2.

In order to ensure that the amplitude and the phase of the impulse responses' taps can be modelled separately, the correlations between them were extracted. The results show that there is no significant correlation between the amplitude and the phase of the impulse responses' taps. KolmogorovSmirnov (KS) tests, with a level of significance of $5 \%$, were performed between, respectively, the coherent component's phases $\phi_{c}$ and the diffuse component's phases $\phi_{d}$, and a uniform distributions between $-\pi$ and $\pi$. The tests results indicate that, in more than $95 \%$ of the cases, there were no significant differences between the samples and the uniform distribution, meaning that $\phi_{c}$ and $\phi_{d}$ can be modelled by random variable taken from a uniform distribution between $-\pi$ and $\pi$. This shows that the amplitude of the impulse responses' tap can be well modelled separately from the phase, and this latter can be added after such as presented in Section 4.

3.2. Correlations. For the coherent component of the impulse responses, no correlation in the delay-domain is experimentally observed for the amplitudes of the different taps. For the diffuse component, two correlations are considered: the correlation between the amplitudes of successive taps of one single impulse response and the correlation between the amplitudes of identical taps of successive impulse responses.

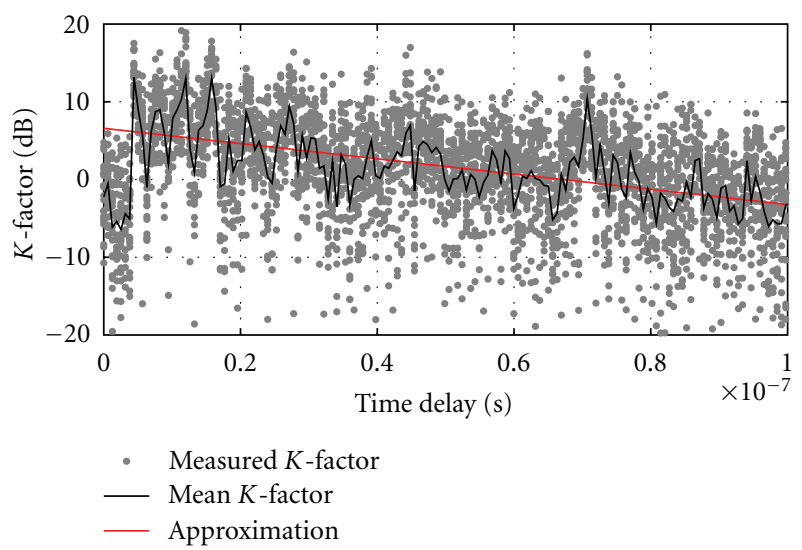

(a) In every scenario, the mean value of the Rice factor $K(\tau)$ presents a linear regression that decreases with the delay

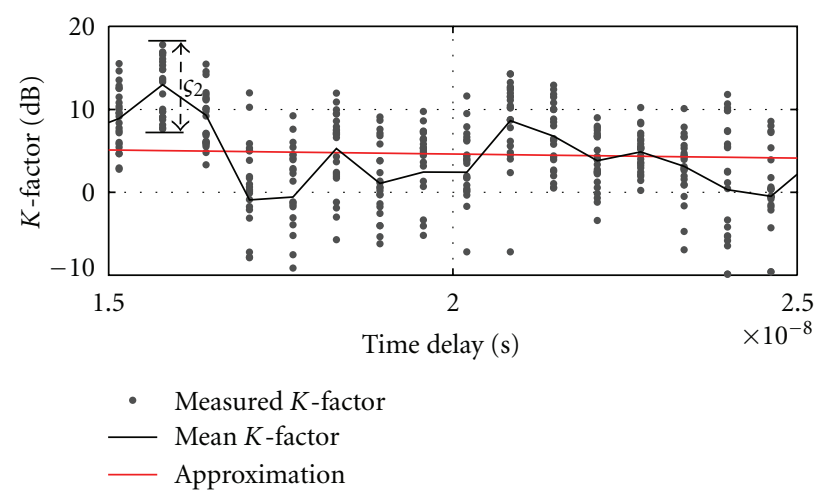

(b) Detailed view of the measured Rice factor $K(\tau)$. The $\mathrm{K}$-factors present, for each delay, a normal distribution (in $\mathrm{dB}$ ) around the mean value with a standard deviation represented by $\varsigma_{2}$

Figure 5: Measured Rice factor $K(\tau)$ of all the measurements' sets for the case with two passengers.

In this paper, the following notation is used for the correlation coefficient $\rho_{X Y}$ :

$$
\rho_{X Y}=\frac{\sum_{i=1}^{N}\left(x_{i}-\bar{x}\right) \cdot\left(y_{i}-\bar{y}\right)}{\sqrt{\sum_{i=1}^{N}\left(x_{i}-\bar{x}\right)^{2}} \cdot \sqrt{\sum_{i=1}^{N}\left(y_{i}-\bar{y}\right)^{2}}},
$$

where $X\left(x_{1}, \ldots, x_{n}\right)$ and $Y\left(y_{1}, \ldots, y_{n}\right)$ are two sets of data with the same length. These sets are defined either in the delay- or in the time-domain.

3.2.1. Delay Correlation of the Diffuse Part. The two data sets are defined as

$$
\begin{gathered}
X=\left|h_{d}\left(t, \tau_{i}\right)\right| \quad \text { for } i=1,2, \ldots, N, \\
Y=\left|h_{d}\left(t, \tau_{j}\right)\right| \quad \text { for } j=i, i+1, \ldots, N,
\end{gathered}
$$

where $N$ represents the maximum number of taps. A typical result for the delay correlation for the diffuse part is drawn in Figure 6. As can be observed, only correlations between adjacent taps (taps $i$ and $j=i+1$ ) have a value higher than 0.5 . For all scenarios, the mean value for this 
TABLE 2: Model parameters.

\begin{tabular}{|c|c|c|c|c|c|c|c|c|c|c|}
\hline \multirow{2}{*}{ Number of passengers } & \multicolumn{2}{|c|}{$1 / B_{d}(\mathrm{~ns})$} & \multicolumn{2}{|c|}{$P_{d}(\mathrm{~dB})$} & \multicolumn{2}{|c|}{$\tau_{d}(\mathrm{~ns})$} & \multirow{2}{*}{$K_{1}(\mathrm{~dB} / \mathrm{sec})$} & \multirow{2}{*}{$K_{0}(\mathrm{~dB})$} & \multirow{2}{*}{$\varsigma_{1}(\mathrm{~dB})$} & \multirow{2}{*}{$\varsigma_{2}(\mathrm{~dB})$} \\
\hline & Mean & Std. & Mean & Std. & Mean & Std. & & & & \\
\hline 1 & 10.73 & 0.41 & -59.32 & 1.19 & 10.73 & 0.63 & $-9.13 e 7$ & 7.43 & 3.45 & 3.5 \\
\hline 2 & 9.25 & 0.35 & -60.03 & 1.23 & 8.83 & 0.63 & $-9.81 e 7$ & 6.58 & 3.2 & 3.6 \\
\hline 3 & 7.23 & 0.28 & -60.45 & 1.33 & 7.57 & 0.63 & $-7.4 e 7$ & 2.35 & 3.03 & 3.45 \\
\hline 4 & 5.61 & 0.23 & -61.79 & 1.54 & 6.94 & 0.63 & $-7.49 e 7$ & 1.71 & 3.15 & 3.6 \\
\hline
\end{tabular}

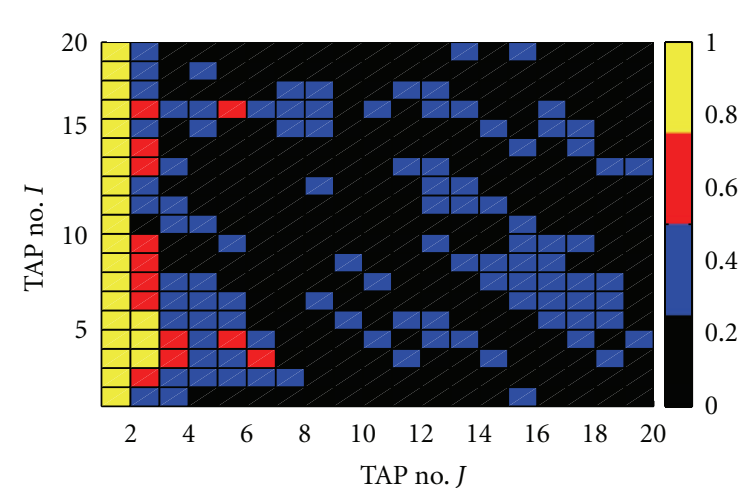

Figure 6: Delay correlation of the diffuse component. Only correlation coefficient with adjacent taps have significant values (above 0.5).

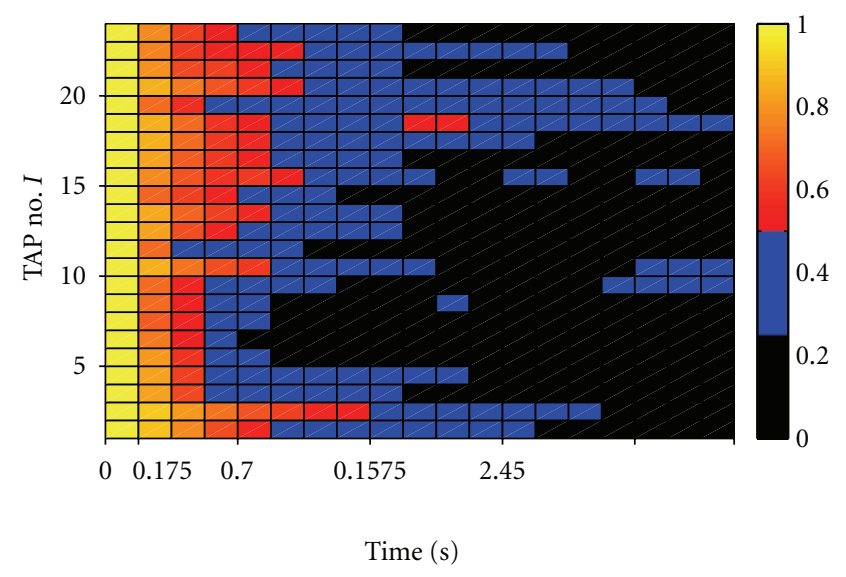

FIGURE 7: Example of the auto-correlation coefficient in the time domain. All those auto-correlations are similar. After $1.575 \mathrm{sec}$, the correlations become insignificant (below 0.5).

correlation coefficient is about $\rho_{d}=0.5$. The other correlation coefficients are smaller and can be neglected. In Sec-tion 4, it will be shown how this can be included into the model.

3.2.2. Temporal Autocorrelation of the Diffuse part. The two data sets are defined as

$$
\begin{aligned}
& X=\left|h_{d}\left(t_{i}, \tau\right)\right| \quad \text { for } i=1,2, \ldots, M, \\
& Y=\left|h_{d}\left(t_{j}, \tau\right)\right| \quad \text { for } j=1,2, \ldots, M,
\end{aligned}
$$

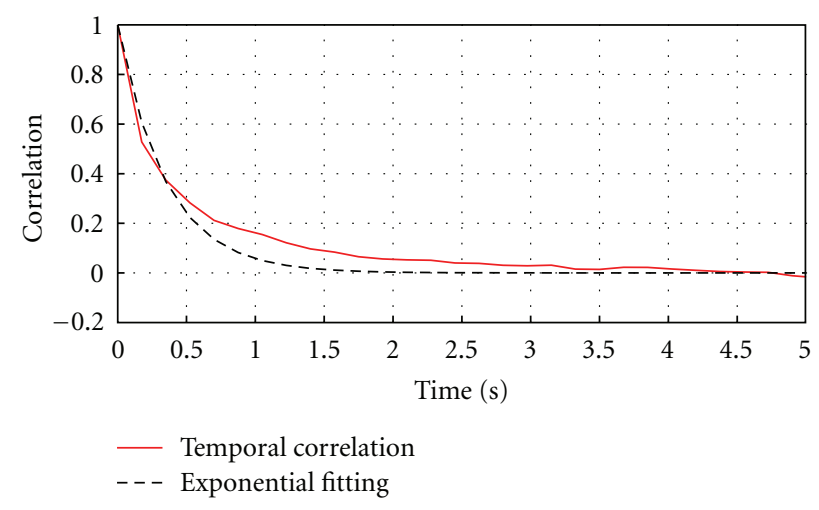

FIGURE 8: The averaged time auto-correlation is fitted with an exponential model.

TABLE 3: Mean and standard deviation of $\gamma$ for each number of passengers.

\begin{tabular}{lcc}
\hline Number of passengers & Mean $(\mathrm{s})$ & Std. $(\mathrm{s})$ \\
\hline 1 & 0.68 & 0.28 \\
2 & 0.57 & 0.15 \\
3 & 0.42 & 0.09 \\
4 & 0.35 & 0.08 \\
\hline
\end{tabular}

where $M$ denotes the maximum number of sweeps (see Section 2.1). A typical example is presented in Figure 7. In this figure, only the first 25 taps are shown, but the behaviour for the other taps is identical. It can be seen that all taps present an autocorrelation in the time domain that can be modelled with an exponential decay. Since all taps show a similar behaviour, the auto-correlation function has been averaged of all taps (within one measurement's set). The averaged correlation is drawn in Figure 8. It is fitted with an exponential model expressed by

$$
\rho_{\Delta t}=\exp \left(-\frac{\Delta t}{\gamma}\right)
$$

The parameter $\gamma$ has been obtained for each scenario by using a least squares estimator on the correlations between 1 and 0.5. Values for $\gamma$ are given in Table 3. As expected, the temporal correlations decrease with the number of passengers. 


\section{Model Implementation}

To summarize the description of the model, a brief summary of the model implementation is presented.

(A) For every sets of channel impulses' responses, for each tap $k$ (with delay $\tau_{k}$ ), the mean $\overline{K\left(\tau_{k}\right)}$ factor is generated using (8) and values given in Table 2.

(B) For each set and for each tap $k$ (with delay $\tau_{k}$ ), the impulse responses can be sequentially generated by the following steps:

(1) From (6) and values given in Table 2, $\psi_{d}\left(\tau_{k}\right)$ is generated. $\sigma_{k}$ is then given by

$$
\sigma_{k}=\sqrt{\frac{\psi_{d}\left(\tau_{k}\right)}{2}}
$$

(2) From the previously generated $\overline{K\left(\tau_{k}\right)}$ and from (9), $K\left(\tau_{k}\right)$ is generated using the values given in Table 2. $\nu_{k}$ is then given by

$$
v_{k}=\sqrt{K\left(\tau_{k}\right) \cdot 2 \sigma_{k}^{2}}
$$

(3) The diffuse component of the impulse responses $h_{d}\left(\tau_{k}, t\right)$ is generated recursively, as explained in the appendix.

(4) The coherent component of the impulse response $h_{c}(\tau)$ is generated

$$
h_{c}\left(\tau_{k}\right)=\left|\nu\left(\tau_{k}\right)\right| e^{-j \phi_{c, k}},
$$

where $\phi_{c, k}$ is random variable with a uniform distribution between $-\pi$ and $\pi$.

(5) Finally, the impulse response is given by the sum of coherent and diffuse components:

$$
h\left(\tau_{k}, t\right)=h_{c}\left(\tau_{k}\right)+h_{d}\left(\tau_{k}, t\right) .
$$

\section{Model Validation}

In order to validate the model presented previously, comparisons between measured and simulated channels were performed. The rms delay spread, the path loss, and the coherence bandwidth were evaluated. The coherence bandwidth is given by the frequency auto-correlation evaluated at 0.5 . Those three parameters are not directly used in the model and can be good metrics in order to validate the model. For each number of passenger, the following parameters were used to generate the channel impulses' responses:

(i) number of sets: 20 ,

(ii) number of impulses' responses per set: 200,

(iii) bandwidth: $1584 \mathrm{MHz}$,

(iv) number of taps: 529 ,

(v) sweep time: $0.175 \mathrm{sec}$.

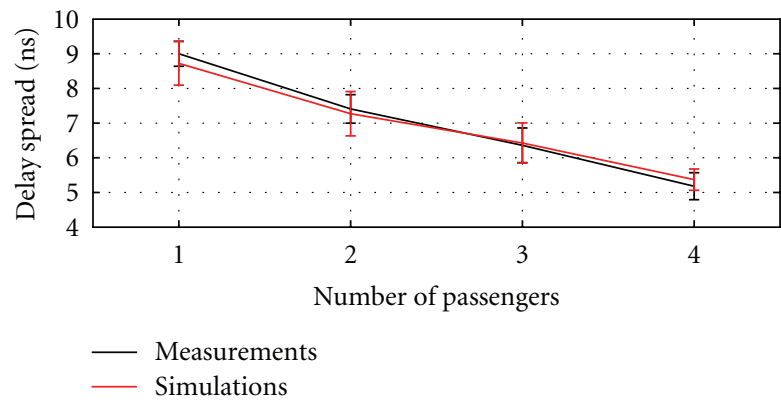

(a) RMS delay spread versus the number of passengers

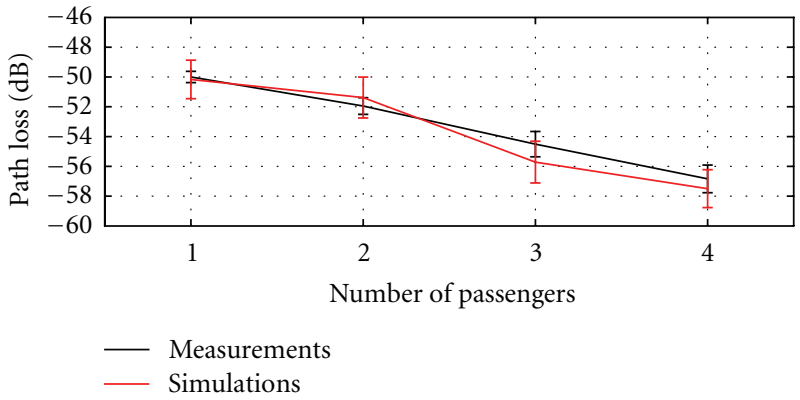

(b) Path loss versus the number of passengers

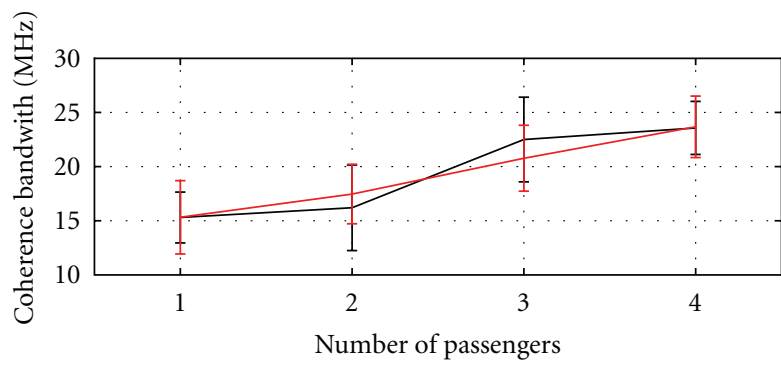

- Measurements

c) Coherence bandwidth versus the number of passengers

Figure 9: Comparisons between simulated and measured channels. The parameters extracted from the simulations do not differ significantly from the measurements. Mean values agree well, and the standard deviations do not differ significantly.

The comparison of the rms delay spread and path loss are, respectively, presented in Figures 9(a) and 9(b). Those parameters were extracted such as for the measurements presented in Section 2.2. As it can be observed, the mean values of the parameters derived from the simulations well match the mean values of the measurements. The standard deviations are a slightly higher in the simulations but the differences are very low. For the measurements, the standard deviations (presented in Table 1) are around $0.4 \mathrm{~ns}$ for the rms delay spread and $0.5 \mathrm{~dB}$ for the path loss. For the simulations, the standard deviations are around $0.6 \mathrm{~ns}$ for the rms delay spread and $1.3 \mathrm{~dB}$ for the path loss, which are very close. In Figure 9(c), the comparison of the coherence bandwidth is presented. The standard deviations are in both cases around $3 \mathrm{MHz}$. 
The results presented previously show that the parameters extracted from the simulations do not differ significantly from the measurements. Some small differences $(<10 \%$ relative error) can be observed between measured and simulated channels but, in general, there is a good matching between the measurements and the model, meaning that this model can be applied for these scenarios.

\section{Conclusion}

In this paper, the UWB wireless communication channel inside a car has been investigated. A channel model, based on the reverberation chambers theory, has been proposed, that splits the channel into a coherent and a diffuse component. A measurement campaign has been conducted to extract parameter values for the model. A preliminary study of the channel's first-and-second order statistics showed that the influence of the environment outside the car is negligible, whereas the influence of the number of passengers significantly changes the propagation environment. The timeand delay-correlations for the diffuse component were also extracted from the measurements. It could be observed that delay-correlation is only significant between adjacent taps, while temporal correlation can last as long as a few hundreds of milliseconds. Finally, the model was successfully validated by comparing generated and measured channel statistics.

\section{Appendix}

As the amplitudes of the diffuse component are Rayleigh distributed, the generation starts with a matrix $\mathbf{R}[N M \times 1]$ given by

$$
\mathbf{R}=\left[\begin{array}{llll}
\mathbf{R}_{\tau_{1}} & \mathbf{R}_{\tau_{2}} & \cdots & \mathbf{R}_{\tau_{\mathrm{N}}}
\end{array}\right]^{T},
$$

where $\mathbf{R}_{\tau_{\mathbf{k}}}$ is a $[1 \times M]$ vector representing the different temporal realizations of tap $k$ at time $t=\left[t_{1} \cdots t_{M}\right]$. Each element of $\mathbf{R}_{\tau_{\mathbf{k}}}$ is a Rayleigh distributed random variables with parameter 1 . The amplitudes, correlated in the delayand time-domain, are given by

$$
\left|h_{d}\left(\tau_{k}, t\right)\right|=C^{1 / 2} \mathrm{R},
$$

where $C^{1 / 2}$ is the correlation matrix. As $C=T \otimes D$, where $T$ represent the temporal auto-correlation matrix and $D$ the delay-correlation matrix, $C^{1 / 2}$ is given by

$$
C^{1 / 2}=T_{c} \otimes D_{c} .
$$

The $c$-indexes represent the Cholesky decomposition of the matrices et $\otimes$ denotes the Kronecker product.

For the auto-correlation matrix $T$, starting from (13) and the parameters from Table 3, the vector $\kappa=$ $\left[1 \rho_{\Delta t_{1}} \rho_{\Delta t_{2}} \cdots \rho_{\Delta t_{M}}\right.$ ] is obtained where $\rho_{\Delta t_{i}}$ represents the auto-correlation coefficient for a time lag $\Delta t_{i}$. The $T_{c}$ matrix is then given by

$$
T_{c}=\left(\operatorname{toep}\left(\kappa, \kappa^{H}\right)\right)^{1 / 2},
$$

where toep represents the Toeplitz operator.
For the delay matrix $D$, only the adjacent taps are correlated. For 2 taps at delay $k$ and $k+1$, the $D_{c}$ matrix is given by

$$
D_{c,(k, k+1)}=\left(\begin{array}{cc}
1 & 0 \\
\rho_{d} & \sqrt{1-\rho_{d}^{2}}
\end{array}\right),
$$

where $\rho_{d}$ is the correlation coefficient. The taps can be correlated recursively from the matrix $D_{c,(k, k+1)}$.

Taking into account (A.3), (A.4) and (A.5), and by adjusting the power level of each tap, it is possible to correlate the matrix $\mathbf{R}$ in the time- and delay-domain by applying the following method:

(i) for tap $k=1$, all the temporal realizations are generated,

$$
h_{d}\left(\tau_{1}, t\right)=\left|\sigma_{1} T_{c} \widetilde{\mathbf{R}}_{\tau_{1}}\right| \cdot \boldsymbol{\Phi}_{1}
$$

(ii) for taps $k=2,3, \ldots, N$, all the temporal realizations are generated

$$
h_{d}\left(\tau_{k}, t\right)=\left|\sigma_{k} T_{c}\left(\rho_{d} \widetilde{\mathbf{R}}_{\tau_{\mathrm{k}-1}}+\sqrt{1-\rho_{d}^{2}} \widetilde{\mathbf{R}}_{\tau_{\mathbf{k}}}\right)\right| \cdot \boldsymbol{\Phi}_{\mathbf{k}},
$$

where $|\cdot|$ denotes the elementwise modulus of a matrix and $\widetilde{\mathbf{R}}_{\tau_{\mathrm{k}}}$ is a $[1 \times M]$ vector representing the different temporal realizations of tap $k$. Each element of $\widetilde{\mathbf{R}}_{\tau_{\mathrm{k}}}$ is a Rayleigh distributed random variables with parameter $1 . \Phi_{\mathrm{k}}$, a $[1 \times M]$ vector representing the phase term, is given by

$$
\boldsymbol{\Phi}_{\mathbf{k}}=\left[\begin{array}{llll}
e^{-j \phi_{d}(k, 1)} & e^{-j \phi_{d}(k, 2)} \cdots e^{-j \phi_{d}(k, M)}
\end{array}\right],
$$

where the phase $\phi_{d}(k, t)$ is distributed according to a uniform distribution between $-\pi$ and $\pi$.

To correlate Rayleigh-distributed random variables, the correlation coefficients $\rho_{d}$ and the coefficients of $T_{c}$ must be adapted according to the method presented in [19].

\section{References}

[1] ECMA-368, "High rate ultra wideband PHY and MAC standard," Tech. Rep., Ecma International, Geneve, Switzerland, 2008.

[2] ECMA-387, "High rate $60 \mathrm{GHz}$ PHY, MAC and HDMI PAL," Tech. Rep., Ecma International, Geneve, Switzerland, 2008.

[3] WirelessHD, "WirelessHD specification overview version 1.0 a," Tech. Rep., WirelessHD, Berlin, Germany, 2009.

[4] Wireless Gigabit Alliance, "WiGig white paper: defining the future of multi-Gigabit Wireless communications," Tech. Rep., Wireless Gigabit Alliance, Beaverton, Ore, USA, 2010.

[5] Y. Katayama, K. Terasaka, K. Higashikaturagi, I. Matunami, and A. Kajiwara, "Ultra-wideband impulse-radio propagation for in-vehicle wireless link," in Proceedings of the 64th IEEE Vehicular Technology Conference, pp. 1-5, Montreal, Quebec, Canada, September 2006.

[6] Y. Nakahata, K. Ono, I. Matsunami, and A. Kajiwara, "Performance evaluation of vehicular Ultra-wideband radio channels," in Proceedings of the 68th IEEE Semi-Annual Vehicular Technology Conference, pp. 1-5, Calgary, Alberta, Canada, September 2008. 
[7] T. Kobayashi, "Measurements and characterization of ultra wideband propagation channels in a passenger-car compartment," in Proceedings of the 9th IEEE International Symposium on Spread Spectrum Techniques and Applications, pp. 228-232, Manaus-Amazon, Brazil, May 2006.

[8] M. Schack, J. Jemai, R. Piesiewicz, R. Geise, I. Schmidt, and T. Kurner, "Measurements and analysis of an in-car UWB channel," in Proceedings of the 67th IEEE Vehicular Technology Conference, pp. 459-463, Singapore, May 2008.

[9] M. Schack, R. Geise, I. Schmidt, R. Piesiewiczll, and T. Kurner, "UWB channel measurements inside different car types," in Proceedings of the 67th IEEE Vehicular Technology Conference, pp. 640-644, May 2008.

[10] M. Schack, M. Jacob, and T. Kürner, "Comparison of in-car UWB and $60 \mathrm{GHz}$ channel measurements," in Proceedings of the 4th European Conference on Antennas and Propagation, pp. 1-5, Barcelona, Spain, June 2010.

[11] M. Peter, R. Felbecker, and W. Keusgen, "Measurementbased investigation of $60 \mathrm{GHz}$ broadband transmission for wireless in-car communication," in Proceedings of the 70th IEEE Vehicular Technology Conference, pp. 1-5, September 2009.

[12] F. Bellens, F. Quitin, F. Horlin, and P. de Doncker, "UWB channel analysis within a moving car," in Proceedings of the 9th International Conference on Intelligent Transport Systems Telecommunications, pp. 681-684, Lille, France, September 2009.

[13] F. Bellens, F. Quitin, F. Horlin, and P. de Doncker, "Channel measurements and MB-OFDM performance inside a driving car," in Proceedings of the International Conference on Electromagnetics in Advanced Applications, pp. 392-395, Torino, Italy, 2009.

[14] N. Czink, A. Richter, E. Bonek, J. P. Nuutinen, and J. Ylitalo, "Including diffuse multipath parameters in MIMO channel models," in Proceedings of the 66th IEEE Vehicular Technology Conference, pp. 874-878, Baltimore, Md, USA, 2007.

[15] O. Delangre, S. Van Roy, P. de Doncker, M. Lienard, and P. Degauque, "Modeling in-vehicle wideband wireless channels using reverberation chamber theory," in Proceedings of the 66th IEEE Vehicular Technology Conference, pp. 2149-2153, 2007.

[16] M. Heddebaut, V. Deniau, and K. Adouane, "In-vehicle WLAN radio-frequency communication characterization," IEEE Transactions on Intelligent Transportation Systems, vol. 5, no. 2, pp. 114-121, 2004.

[17] G. D. Durgin, Space-Time Wireless Channels, Communications Engineering and Emerging Technologies Series, Prentice Hall, 2003.

[18] C. L. Holloway, D. A. Hill, J. M. Ladbury, P. F. Wilson, G. K. Koepke, and J. Coder, "On the use of reverberation chambers to simulate a rician radio environment for the testing of wireless devices," IEEE Transactions on Antennas and Propagation, vol. 54, no. 11, pp. 3167-3177, 2006.

[19] C. A. G. D. de Leon, M. C. Bean, and J. S. Garcia, "On the generation of correlated Rayleigh envelopes for representing the variant behavior of the indoor radio propagation channel," in Proceedings of the 15th IEEE International Symposium on Personal, Indoor and Mobile Radio Communications, vol. 4, pp. 2757-2761, 2004. 

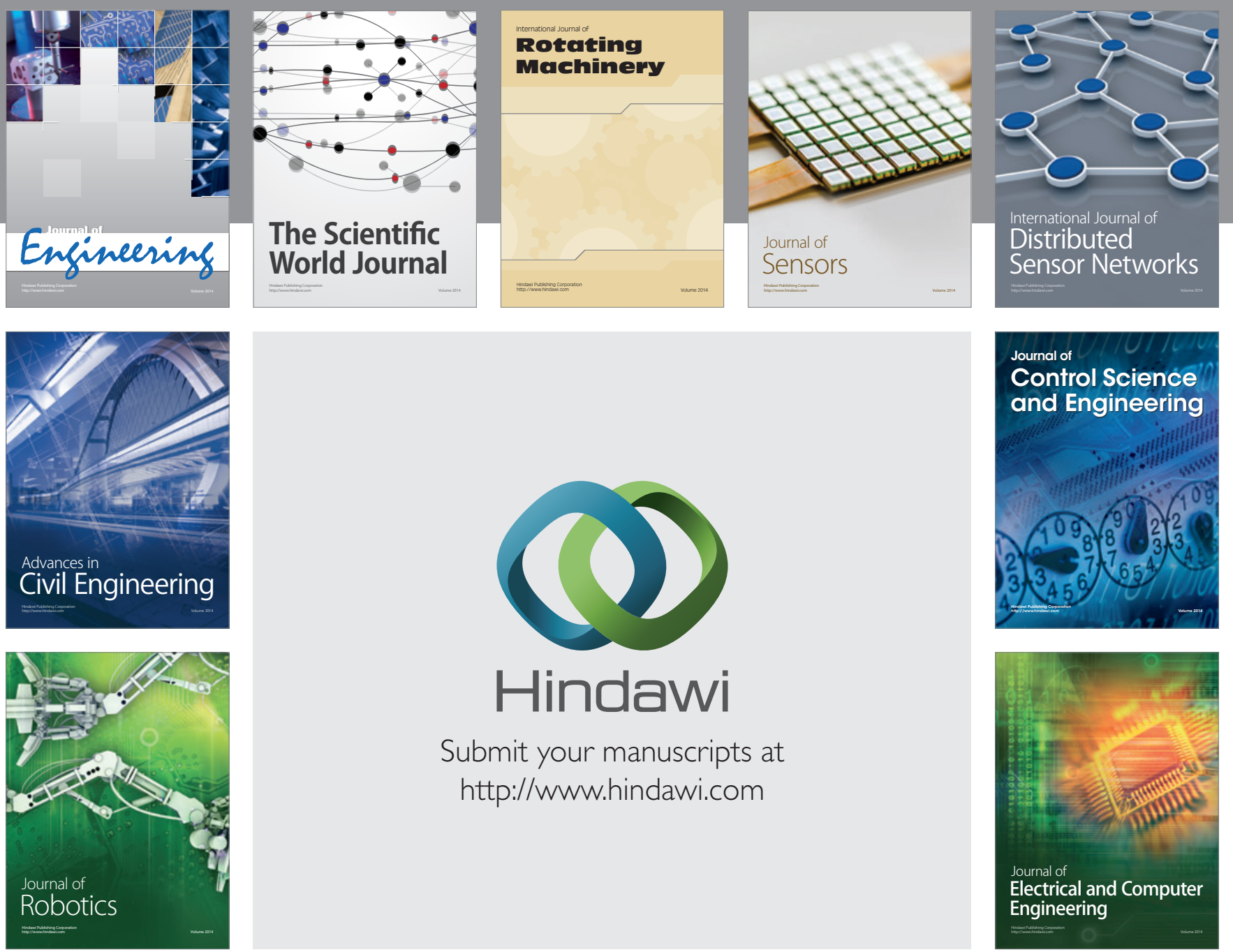

Submit your manuscripts at

http://www.hindawi.com
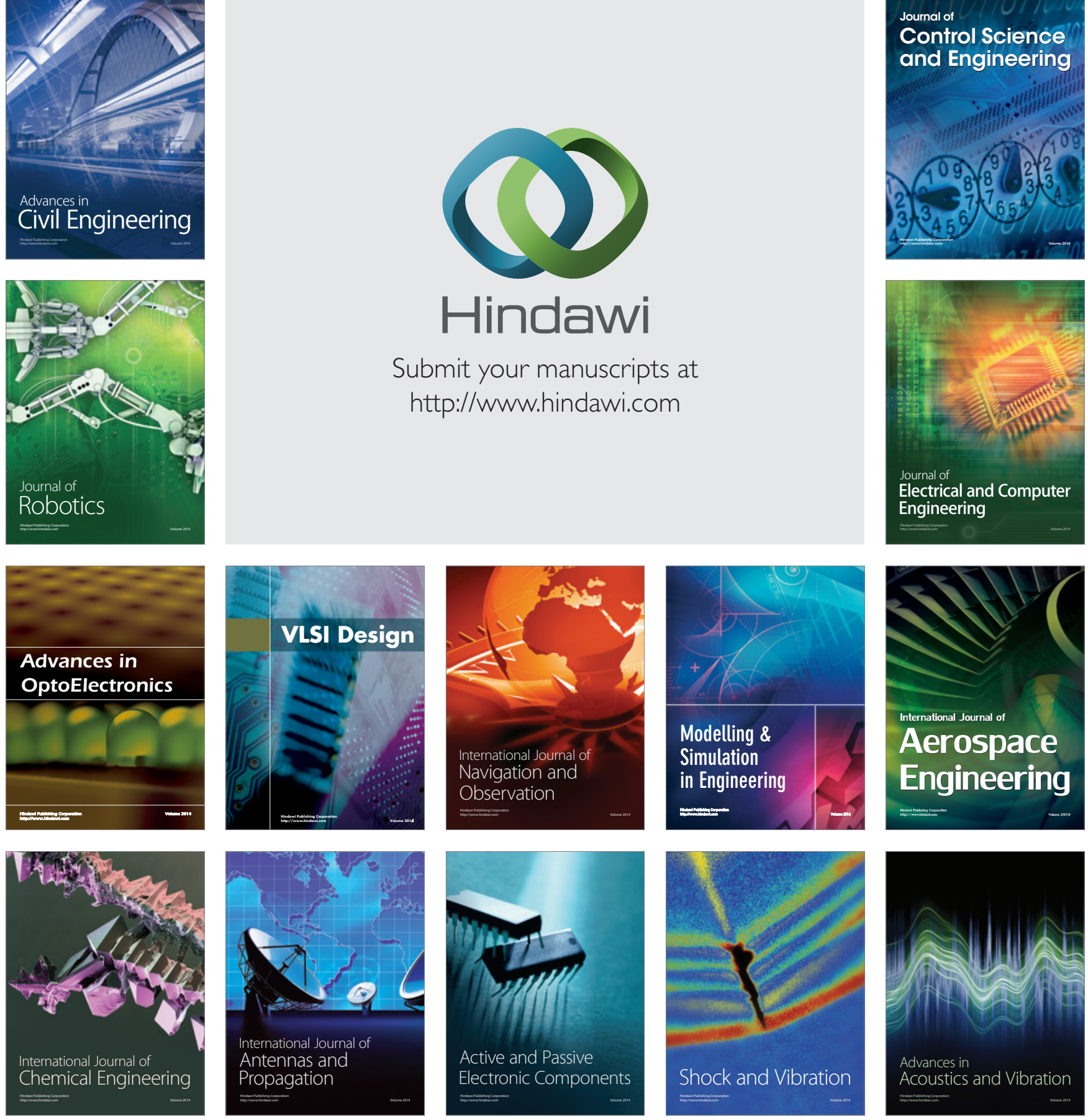